\title{
JOURNAL SCAN
}

\section{Journal Scan}

\author{
U. C. Ojha
}

Senior Specialist \& Head - Department of Respiratory Medicine, E.S.I Hospital, New Delhi

Indian J Sleep Med 2012; 7.2, 62-68

1.Am J Respir Crit Care Med Vol 184. pp 1140-1146, 2011

\section{Burnout in ICU Caregivers A Multicenter Study of Factors Associated to Centers}

\section{Paolo Merlani', Me 'lanie Verdon', Adrian Businger ${ }^{2}$, Guido Domenighetti ${ }^{3}$, Hans Pargger', Bara Ricou', and the STRESI' Group*}

Service of Intensive Care, Department of Anesthesiology, Pharmacology, and Intensive Care, Geneva University Hospitals and the University of Geneva, Geneva; 2Division of Operative Intensive Care, Department of Anesthesia and Intensive Care, University Hospital of Basel, Basel; and ${ }^{3}$ Multidisciplinary Intensive Care, Regional Hospital of Locarno, Locarno, Switzerland

Rationale: The stressful work environment of ICUs can lead to burnout. Burnout can impact on the welfare and performance of caregivers, and may lead them to resign their job. The shortage of ICU caregivers is becoming a real threat for health care leaders.

Objectives: To investigate the factors associated with burnout on a national level in order to determine potential important factors.

Methods: Prospective, multicenter, observational survey of all caregivers from 74 of the 92 Swiss ICUs, measuring the prevalence of burnout among the caregivers and the pre-specified center-, patient and caregiver-related factors influencing its prevalence.

\section{Address for correspondence}

Dr. U. C. Ojha

Senior Consultant and Head of the Department Specialist \& Head, Dept of Respiratory Medicine E.S.I. Hospital, Basaidarapur, New Delhi Email: ucojha@rediffmail.com

Indian Journal of Sleep Medicine (IJSM), Vol. 7, No. 2, 2012
Measurements and Main Results: Out of the 4322 questionnaires distributed from March 2006 to April 2007, $3052(71 \%)$ were returned, with a response rate of $72 \%$ by center, $69 \%$ from nurse assistants, $73 \%$ from nurses and $69 \%$ from physicians. A high proportion of female nurses among the team was associated with a decreased individual risk of high burnout (OR 0.98, 95\% CI:0.97-0.99 for every \%). The caregiver-related factors associated with a high risk of burnout were being a nurseassistant, being a male, having no children and being under 40 years old.

Conclusions: The findings of this study seem to open a new frontier concerning burnout in ICUs, highlighting the importance of team composition. Our results should be confirmed in a prospective multicenter, multinational study. Whether our results can be exported to other medical settings where team-working is pivotal remains to be investigated.

Keywords: critical care; job satisfaction; multicenter; intensive care unit management; intensive care unit organization

2. Conf Proc IEEE Eng Med Biol Soc. 2011;2011:831922.

Developing quantitative physiological phenotypes of sleep apnea for epidemiological studies.

\section{Kirkness JP, McGinley BM, Sgambati FP, Patil SP, Smith PL, Schwartz AR, Schneider H.}

Johns Hopkins Sleep Disorders Center, Division of Pulmonary Medicine, Johns, Hopkins University, Baltimore, MD, USA.

Existing physiological databases have not been sufficiently detailed to provide relevant and important information 
for characterizing the pathophysiology of obstructive sleep apnea. Critical collapsing pressure $(\mathrm{P}(\mathrm{CRIT}))$ is a standard method for determining upper airway patency during sleep, however is labor intensive and prohibits large-scale studies. Based on previously published data indicating R(US) does not significantly vary between groups, our aim was to develop an approach to estimate the $\mathrm{P}(\mathrm{CRIT})$ from airflow at atmospheric pressure $(\mathrm{V}(\mathrm{atm}))$. In a dataset of 126 subjects, where P(CRIT) and R(US) were measured using standard techniques. We then determined the minimum sample size required to estimate the R(US) mean and variance by utilizing a bootstrap procedure ( 30 times for $n=3$ to 126 ). We first estimated the minimum number of subjects needed for obtaining a group for a two-tailed $(\mathrm{z}=1.96)$ standard error for $\mathrm{R}(\mathrm{US})$ in the population. Then in 75 individuals, quantitative estimates of airflow were obtained at atmospheric pressure. Using the estimated R(US) and atmospheric, we determined an estimated P(CRIT) (a $\mathrm{P}(\mathrm{CRIT}))$. Bland-Altman plots were generated to determine the agreement between the measured $\mathrm{P}$ (CRIT) and $\mathrm{P}(\mathrm{CRIT})$. For the entire population the mean \pm SEM R(US) was $23 \pm 1 \mathrm{cmH}(2) \mathrm{O} / \mathrm{L} / \mathrm{s}( \pm 95 \% \mathrm{CI}: 21$, 25). -40 subjects represent the minimum sample required to estimate the population variance within \pm 2 SEM. In the subsample with atmospheric flow measurements, a linear regression model (aP(CRIT) $[\mathrm{cmH}(2) \mathrm{O}]=\mathrm{V}(@ \mathrm{PN})$ $[\mathrm{L} / \mathrm{s}] \mathrm{x}-23[\mathrm{cmH}(2) \mathrm{O} / \mathrm{L} / \mathrm{s}])$, ${ }^{\mathrm{P}} \mathrm{P}(\mathrm{CRIT})$ ranged from 0 to $9.6 \mathrm{cmH}(2) \mathrm{O}$. In the Bland-Altman analysis there was no mean difference between the measured $\mathrm{P}(\mathrm{CRIT})$ and aP(CRIT) $(-0.01 \mathrm{cmH}(2) \mathrm{O} ; \mathrm{p}=0.8)$ with upper and lower limits of agreement at $\pm 2.3 \mathrm{cmH}(2) \mathrm{O}$. The variance of upstream resistance approaches a constant value in groups with approximately 40 subjects. Utilizing a fixed up-stream resistance to estimate $\mathrm{P}(\mathrm{CRIT})$ from the airflow at atmospheric pressure agrees with the measured values. These data suggest that measurements of quantitative airflow during standard polysomnography can be used to determine upper airway properties in large cohorts.
3. Braz J Otorhinolaryngol. 2011 Jun;77(3):348-55.

\section{Polymorphisms in the 5-HTR2A gene related to obstructive sleep apnea syndrome.}

[Article in English, Portuguese]

\section{Piatto VB, Carvalho TB, De Marchi NS, Molina FD, Maniglia JV.}

Otorhinolaryngology and Head \& Neck Surgery Department, FAMERP. vbpiatto@gmail.com

Obstructive sleep apnea syndrome (OSAS) is one of the most complex disorders of sleep; it involves several genetic factors that contribute to the phenotype.

Serotonin (5-HT) regulates a variety of visceral and physiological functions, including sleep. Gene 5-HTR2A polymorphisms may change the transcription of several receptors in the serotoninergic system, thereby contributing to OSAS.

AIM: To investigate the prevalence of T102C and $1438 \mathrm{G} / \mathrm{A}$ polymorphisms in the 5-HTR2A gene of patients with and without OSAS.

MATERIAL AND METHOD: A molecular study of 100 index-cases and 100 controls of both genders. DNA was extracted from blood leukocytes samples and the regions that enclose both polymorphisms were amplified with PCR-RFLP.

STUDY DESIGN: A cross-sectional case study.

RESULTS: There was a significant prevalence of males in index cases compared to controls $(\mathrm{p}<0.0001)$. No significant genotypic differences between cases and controls were found in T102C polymorphisms $(p=1.000)$. There were significant differences between the AA genotype of $-1438 \mathrm{G} / \mathrm{A}$ polymorphisms and patients with OSAS (OR:2.3; CI95\%:1.20-4.38, $\mathrm{p}=0.01)$.

CONCLUSION: Serotonergic mechanisms may be related to OSAS. There were no differences in the prevalence of $\mathrm{T} 102 \mathrm{C}$ polymorphisms in patients with OSAS and the control group. There is evidence of an association between the $-1438 \mathrm{G} / \mathrm{A}$ polymorphism and OSAS. 
4. Sleep. 2011 Jul 1;34(7):957-64.

\section{Heritability and mortality risk of insomnia-related symptoms: a genetic epidemiologic study in a population- based twin cohort.}

\section{Hublin C, Partinen M, Koskenvuo M, Kaprio J.}

Finnish Institute of Occupational Health, Helsinki, Finland. christer.hublin@ttl.fi

STUDY OBJECTIVES: Our aim was to estimate heritability in phenotypic insomnia and the association between insomnia and mortality.

DESIGN: Representative follow-up study.

PARTICIPANTS: 1990 survey of the Finnish Twin Cohort ( $\mathrm{N}=12502$ adults; 1554 monozygotic and 2991 dizygotic twin pairs).

MEASUREMENTS: Current insomnia-related symptoms (insomnia in general, difficulty in initiating sleep, sleep latency, nocturnal awakening, early morning awakening, and non-restorative sleep assessed in the morning and during the day) were asked. Latent class analysis was used to classify subjects into different sleep quality classes. Quantitative genetic modelling was used to estimate heritability. Mortality data was obtained from national registers until end of April 2009.

RESULTS: The heritability estimates of each symptom were similar in both genders varying from 34\% (early morning awakening) to $45 \%$ (nocturnal awakening). The most parsimonious latent class analysis produced 3 classes: good sleepers (48\%), average sleepers (up to weekly symptoms, $40 \%$ ), and poor sleepers (symptoms daily or almost daily, $12 \%)$. The heritability estimate for the cluster was $46 \%$ (95\% confidence interval $41 \%$ to $50 \%)$. In a model adjusted for smoking, BMI, and depressive symptoms, the all-cause mortality of poor sleepers was elevated (excess mortality 55\% in men and $51 \%$ in women). Further adjustment for sleep length, use of sleep promoting medications, and sleep apnearelated symptoms did not change the results.

CONCLUSIONS: Insomnia-related symptoms were common in both genders. The symptoms and their clusters showed moderate heritability estimates. A significant association was found between poor sleep and risk of mortality, especially in those with somatic disease.
5. Expert Rev Respir Med. 2011 Jun;5(3):377-93.

\section{Asthma-related comorbidities.}

\section{Boulet LP, Boulay MÈ.}

Centre de Recherche de l'Institut Universitaire de Cardiologie et de Pneumologie, de Québec, 2725, Chemin Sainte-Foy, QC, G1V 4G5, Canada. lpboulet@med.ulaval.ca

Asthma is often associated with various comorbidities. The most frequently reported asthma comorbid conditions include rhinitis, sinusitis, gastroesophageal reflux disease, obstructive sleep apnea, hormonal disorders and psychopathologies. These conditions may, first: share a common pathophysiological mechanism with asthma; second: influence asthma control, its phenotype and response to treatment; and third: be more prevalent in asthmatic patients but without obvious influence on this disease. For many of these, how they interact with asthma remains to be further documented, particularly for severe asthma. If considered relevant, they should, however, be treated appropriately. Further research is needed on the relationships between these conditions and asthma.

6. Expert Rev Neurother. 2011 Apr;11(4):565-77.

\section{Sleep disturbances in children with attention-deficit/hyperactivity disorder.}

\section{Spruyt K, Gozal D.}

Department of Pediatrics, Comer Children's Hospital, Pritzker School of Medicine, The University of Chicago, Chicago, Illinois 60637, USA.

In this article, we advocate the need for better understanding and treatment of children exhibiting inattentive, hyperactive, impulsive behaviors, by in-depth questioning on sleepiness, sleep-disordered breathing or problematic behaviors at bedtime, during the night and upon awakening, as well as night-to-night sleep duration variability. The relationships between sleep andattentiondeficit/hyperactivity disorder (ADHD) are complex and are routinely overlooked by practitioners. Motricity and somnolence, the most consistent complaints and objectively measured sleep problems in children with 
ADHD, may develop as a consequence of multidirectional and multifactorial pathways. Therefore, subjectively perceived or reported restless sleep should be evaluated with specific attention to restless legs syndrome or periodic limb movement disorder, and awakenings should be queried with regard to parasomnias, dyssomnias and sleep-disordered breathing. Sleep hygiene logs detailing sleep onset and offset quantitatively, as well as qualitatively, are required. More studies in children with ADHD are needed to reveal the 24-h phenotype, or its sleep comorbidities.

7. Eur Respir J. 2011 Jul;38(1):98-105. Epub 2011 Mar 15.

\section{Residual sleepiness in obstructive sleep apnoea: phenotype and related symptoms.}

\section{Vernet C, Redolfi S, Attali V, Konofal E, Brion A, Frija-Orvoen E, Pottier M, Similowski T, Arnulf I.}

Sleep Disorders Unit, UMR 975, National Reference Centre for Narcolepsy and Hypersomnia, Paris, France.

The characteristics of residual excessive sleepiness (RES), defined by an Epworth score $>10$ in adequately treated apnoeic patients, are unknown. 40 apnoeic patients, with $(n=20)$ and without $(n=20)$ RES, and 20 healthy controls underwent clinical interviews, cognitive and biological tests, polysomnography, a multiple sleep latency test, and 24-h sleep monitoring. The marked subjective sleepiness in the RES group (mean \pm sd score $16.4 \pm 3$ ) contrasted with moderately abnormal objective measures of sleepiness $(90 \%$ of patients with RES had daytime sleep latencies $>8 \mathrm{~min}$ ). Compared with patients without RES, the patients with RES had more fatigue, lower stage N3 percentages, more periodic leg movements (without arousals), lower mean sleep latencies and longer daytime sleep periods. Most neuropsychological dimensions (morning headaches, memory complaints, spatial memory, inattention, apathy, depression, anxiety and lack of self-confidence) were not different between patients with and without RES, but gradually altered from controls to apnoeic patients without and then with RES. RES in apnoeic patients differs markedly from sleepiness in central hypersomnia. The association between RES, periodic leg movements, apathy and depressive mood parallels the post-hypoxic lesions in noradrenaline, dopamine and serotonin systems in animals exposed to intermittent hypoxia.

8. Sleep. 2011 Feb 1;34(2):165-70.

\section{Daytime sleepiness and REM sleep characteristics in myotonic dystrophy: a case-control study.}

Yu H, Laberge L, Jaussent I, Bayard S, Scholtz S, Raoul M, Pages M, Dauvilliers Y.

Service de Neurologie, Hôpital Gui-de-Chauliac, 80 avenue Augustin Fliche, Montpellier cedex 5, France.

STUDY OBJECTIVES: Excessive daytime sleepiness (EDS) and high daytime REM sleep pressure are important sleep features of myotonic dystrophy (DM1). Small and uncontrolled studies have focused on EDS phenotype; none have focused on nocturnal REM sleep characteristics in DM1. Our objectives were to compare polysomnographic and multiple sleep latency test (MSLT) parameters, and both tonic and phasic components of REM sleep between DM1 and controls.

DESIGN AND PATIENTS: Forty consecutive DM1 patients and 40 sex- and age-matched controls were included. All subjects underwent overnight polysomnography followed by a MSLT.

RESULTS: About $80 \%$ of DM1 patients complained of EDS through clinical interview: $31.4 \%$ had Epworth scores $>10$, and $12.5 \%$ had objective sleepiness (latency $<8 \mathrm{~min}$ ). Higher apnea and central apnea indexes, and a greater proportion of subjects with severe apnea/ hypopnea syndrome were found in DM1. The number of SOREMP differed between DM1 and controls, one and two SOREMPs being present in $47.5 \%$ and $32.5 \%$, and one control had one SOREMP. Higher percentages of slow wave sleep and REM sleep were found in DM1. DM1 patients had significantly more PLMW, PLMS in both NREM and REM sleep, and PLMS-associated microarousals. Higher REM density was found in DM1 with similar tendencies for either REM sleep without atonia or phasic EMG activity.

CONCLUSIONS: This is the first case-control sleep study in DM1 to demonstrate higher frequency of daytime sleepiness and abnormalities in REM sleep 
regulation, with an increased daytime and nighttime REM sleep propensity, REM density, and PLMS. These data suggest a primary central sleep regulation dysfunction in DM1.

\section{Pac Symp Biocomput. 2011:14-25.}

\section{Systems biology analyses of gene expression and genome wide association study data in obstructive sleep apnea.}

Liu Y, Patel S, Nibbe R, Maxwell S, Chowdhury SA, Koyuturk M, Zhu X, Larkin EK, Buxbaum SG, Punjabi NM, Gharib SA, Redline S, Chance MR.

Center for Proteomics \& Bioinformatics, Case Western Reserve University (CWRU), Cleveland, Ohio, 44106, USA.yx1442@case.edu.

The precise molecular etiology of obstructive sleep apnea (OSA) is unknown; however recent research indicates that several interconnected aberrant pathways and molecular abnormalities are contributors to OSA. Identifying the genes and pathways associated with OSA can help to expand our understanding of the risk factors for the disease as well as provide new avenues for potential treatment. Towards these goals, we have integrated relevant high dimensional data from various sources, such as genome-wide expression data (microarray), protein-protein interaction (PPI) data and results from genome-wide association studies (GWAS) in order to define sub-network elements that connect some of the known pathways related to the disease as well as define novel regulatory modules related to OSA. Two distinct approaches are applied to identify sub-networks significantly associated with OSA. In the first case we used a biased approach based on sixty genes/proteins with known associations with sleep disorders and/or metabolic disease to seed a search using commercial software to discover networks associated with disease followed by information theoretic (mutual information) scoring of the sub-networks. In the second case we used an unbiased approach and generated an interactome constructed from publicly available gene expression profiles and PPI databases, followed by scoring of the network with p-values from GWAS data derived from OSA patients to uncover sub-networks significant for the disease phenotype. A comparison of the approaches reveals a number of proteins that have been previously known to be associated with OSA or sleep. In addition, our results indicate a novel association of Phosphoinositide 3-kinase, the STAT family of proteins and its related pathways with OSA.

10. Ann Hematol. 2011 Feb;90(2):145-50.

\section{Cost-effectiveness of adenotonsillectomy in reducing obstructive sleep apnea, cerebrovascular ischemia, vaso-occlusive pain, and ACS episodes in pediatric sickle cell disease.}

\section{Tripathi A, Jerrell JM, Stallworth JR.}

Department of Epidemiology and Biostatistics, University of South Carolina Arnold School of Public Health, Columbia, SC, USA.

In children with sickle cell disease (SCD), adenotonsillar hypertrophy or recurrent tonsillitis are frequently linked with an increased risk of obstructive sleep apnea, cerebrovascular ischemia, or frequent pain episodes and often require an adenoidectomy and/or tonsillectomy. Interventions designed to prevent these complications, control vaso-occlusive pain episodes, and avoid hospitalizations may reduce the significant personal and economic burden of SCD. This study compares episode recurrence and treatment costs for cerebrovascular ischemia, vaso-occlusive pain, acute chest syndrome (ACS), and obstructive sleep apnea in children who had an adenotonsillectomy (A/T surgery, $\mathrm{N}=256 ; 11.7 \%$ ) and a matched cohort of those who did not $(\mathrm{N}=512$; $23.3 \%$ ) from a cohort of 2,194 children and adolescents with SCD from South Carolina's Medicaid system. A/T surgery was associated with a significantly reduced rate of visits over time for obstructive sleep apnea and cerebrovascular ischemia (e.g., stroke, transient ischemic attacks), but not with any change in the rate of visits for vaso-occlusive pain or ACS/pneumonia visits. The rate of mean acute (emergency and inpatient) service costs was significantly decreasing over time after an increase about the time the A/T surgery was performed. The costeffectiveness of adenoidectomy and/or tonsillectomy for treating obstructive sleep apnea and preventing cerebrovascular ischemia without increasing vasoocclusive pain episodes or long-term acute service costs 
in routine clinical practice settings was demonstrated. The matched control group of SCD patients without A/ $\mathrm{T}$ surgery contained more patients with severe vasoocclusive pain episodes, ACS visits, and higher mean total costs over time and appears to represent a different phenotype of children with SCD.

\section{Acta Odontol Latinoam. 2010;23(1):13-9.}

\section{Polymorphisms of the noggin gene and mandibular micrognathia: a first approximation.}

\section{Gutiérrez SJ, Gómez M, Rey JA, Ochoa M, Gutiérrez SM, Prieto JC.}

Dental Research Center, School of Dentistry, Javeriana University, Bogotá, Colombia. s.gutierrez@javeriana.edu.co

Mandibular micrognathia is a deficiency in mandibular growth that prevents tooth contact during mastication, interferes with phonation and even causes sleep apnea. Studies show that mutant mice for chd (chordin) and nog (noggin) genes, which are modulators of the Bone Morphogenic Protein (BMP), had mandibular defects ranging from mandibular hypoplasia to micrognathia and agnathia. The human NOG gene was the first BMP antagonist identified and it is essential for various late events in mandibular development, which require modulation of the BMP activity. The aim of this work was to determine the presence of NOG gene polymorphisms in families with mandibular micrognathia and analyze its phenotype. Four families with mandibular micrognathia were included in this study. Blood samples were taken from the participating individuals through venipuncture and DNA was extracted. The fragments of interest were amplified using the Polymerase Chain Reaction (PCR) and the Single Nucleotide Polymorphisms (SNPs) of the NOG gene reported in the NCBI data base were analyzed through direct sequencing. The SNP rs1348322 was present in homozygote form in the subjects from all the families, where Cytosine is changed to Adenine in position 112 of the exon of the NOG gene. The SNP rs1236187 did not show any clear result. This result suggests that there may be population polymorphism, or markers that are seldom polymorphic for our population. It is therefore necessary to continue with the search for the relationship of the NOG gene with mandibular micrognathia.
12. Sleep Breath. 2011 Sep;15(3):403-8.

\section{Non-synonymous polymorphism in the neuropeptide $S$ precursor gene and sleep apnea.}

\section{Sánchez-de-la-Torre M, Pierola J, Vidal C, Barceló A, de la Peña M, Hussain Z, Capote F, Durán J, Agustí AG, de Lecea L, Torres G, Esquinas C, Martinez M, Barbé F.}

Respiratory Diseases Research Unit, IRB Lleida, Lleida, Spain.

BACKGROUND: Obstructive sleep apnea syndrome (OSAS) is a complex disease with a strong genetic basis. One of the primary molecular domains affected by OSAS is sympathetic activity. Neuropeptide S (NPS) plays an important role in the regulation of the sleep-wakefulness cycle, anxiety states, and daytime sleepiness. It is important to study neuropeptides related to sympathetic activity regulation and how their function could be modified by genetic variants affecting the expression of these molecules.

OBJECTIVES: We investigated the association of the non-synonymous polymorphism rs4751440 in the NPS precursor gene with OSAS and certain variables related to OSAS (daytime sleepiness, body mass index (BMI), insulin resistance, and blood pressure). This polymorphism causes an amino acid substitution in exon 3 of the human NPS precursor gene.

PATIENTS AND METHODS: We included 253 OSAS patients and 70 healthy subjects. Genotyping was done by polymerase chain reaction using specific flanking primers and agarose gel electrophoresis. Daytime sleepiness, BMI, plasma levels of high-density lipoprotein, glucose, total cholesterol, insulin, triglycerides, and the homeostasis model assessment index were also determined.

RESULTS: A similar genotypic and allelic distribution was found in OSAS patients and controls. The risk of OSAS was not associated with the rs4751440 polymorphism. There was no significant interaction between daytime sleepiness or metabolic variables and the rs4751440 polymorphism.

CONCLUSION: Genotypic and allelic frequency distribution of the rs 4751440 polymorphism was similar in OSAS patients and controls. In this population-based 
study, we could not show a significant association between rs4751440 polymorphism and susceptibility to OSAS or certain phenotypes related to OSAS (daytime sleepiness, BMI, systolic blood pressure, and insulin resistance) with the exception of diastolic blood pressure. 